Patients (or Participants) Skiers and snowboarders who played in Niseko were subjected in this study.

Interventions (or Assessment of Risk Factors) Interview survey was carried out in Niseko. We investigated the number of injuries in Niseko ski resort and the situation of severe ski accidents in Japan.

Main Outcome Measurements The type, part, and cause of injury and the effort for preventing accidents in Niseko ski resort.

Results About 50 severe backcountry skiing injuries occur every year in Japan, and 20 of them were fatal. Severe injuries means head injury, back injury and suffocation. During 1985 and 2000, there were 9 skiing fatalities in Niseko every year. All fatalities were caused by avalanches. 8 fatalities were backcountry skiing. These Niseko ski resorts prohibited out of bounds skiing ('Niseko Rule'). The Niseko Rule was promulgated in 2001. No fatalities have occurred since 2001 for backcountry skiing. Only $20 \%$ of skiers wear helmets in Japan, however approximately over $60 \%$ people wear the helmet.

Conclusions The unique point of the Niseko Rule is that Niseko resorts and the local community respect the freedom of mountain users and place a strong emphasis on the sage usage of the mountain. Ski Patrol checks the conditions of each ski resort for the boundaries of the Niseko Rule. Niseko resorts are increasing the rate of wear the skiing helmets. It was influenced by foreign tourist. It will contribute to skiing safety.

\section{PREVENTING INTENTIONAL INJURY (HARASSMENT AND ABUSE) IN SPORT: ASSESSING ATHLETES' KNOWLEDGE, ATTITUDES, AND BELIEFS ABOUT THEIR HUMAN RIGHTS IN THE OLYMPIC AND PARALYMPIC MOVEMENTS}

${ }^{1}$ Demetri Goutos, ${ }^{2}$ Sheree Bekker, ${ }^{3}$ Natalie Galea, ${ }^{4}$ Katharina Grimm, ${ }^{5}$ Margo Mountioy, ${ }^{1}$ Yetsa Tuakli-Wosornu. 'Sports Equity Lab in association with Yale University, Yale School of Public Health, New Haven, USA; 'Department for Health, University of Bath, Bath, UK; ${ }^{3}$ Australian Human Rights Institute, UNSW Sydney, Sydney, Australia; ${ }^{4}$ ShePower Sport, London, UK; ${ }^{5}$ McMaster University, Hamilton, Canada

\subsection{6/bjsports-2021-IOC.360}

Background Egregious cases of athlete abuse continue to demonstrate the link between human rights and sport. However, it is unclear if athletes see themselves as rights-holders in the sports context, and what this means for preventing intentional injury (harassment and abuse).

Objective Assess athletes' knowledge, attitudes, and beliefs about their rights as articulated by the International Olympic Committee Athletes Rights Declaration.

Design Cross-sectional web-based survey.

Setting Web-based communication and social media platforms used to assess elite athletes.

Participants 645 athletes participating in the Olympic and Paralympic movements, representing 70 countries.

Intervention Athletes and sport organizations distributed the survey to Para and non-disabled athletes. Data was collected from February to September 2020.

Main Outcomes Part 1 (Knowledge construct) used yes/no questions to test athletes' knowledge of five rights from the IOC Declaration. Part 2 (Attitudes/Beliefs construct) used Likert scales to test agreement with eight plain language right statements. Correlation analysis examined the relationship between athletes' knowledge and their attitudes/beliefs. Demographic data were analyzed for trends.

Results Athletes' knowledge of the right to protect their name, image, and performance, as well as their right to unbiased redress for rights violations, was weakest. There were varied perceptions about freedom of expression and how acceptable 'pressure' from coaches and teammates was. There was low correlation between athletes' knowledge of a right and their positive attitudes and beliefs about embodying that right in sport-specific scenarios. Gender and union membership significantly impacted athletes' rights-experience.

Conclusions Athletes have incomplete knowledge and mixed perceptions of their rights in the sports realm. Furthermore, knowledge of their rights does not guarantee athletes' confidence in defending those rights during real-life sport experiences. To prevent athlete harassment and abuse, a culture change is required in sport. This cannot happen until athletes' rights are clearly understood and guaranteed by all.

\section{ASSESSMENT OF RISK FACTORS ASSOCIATED WITH INCLUSION IN THE US CENTER FOR SAFESPORT CENTRALIZED DISCIPLINARY DATABASE}

${ }^{1,2}$ Nida Naushad, ${ }^{3}$ Rebecca Fix ${ }^{4}$ Allison Wagner, ${ }^{5}$ Demetri Goutos, ${ }^{1,2}$ Yetsa Tuak Wosornu. ' Y Yale School of Medicine, New Haven, USA; ${ }^{2}$ Yale School of Public Health, New Haven, USA; ${ }^{3}$ Johns Hopkins Bloomberg School of Public Health, Baltimore, USA; ${ }^{4}$ Erasmus Mundus, Swansea, USA; ${ }^{5}$ Sports Equity Lab in association with Yale University, Yale School of Public Health, New Haven, USA

\subsection{6/bjsports-2021-IOC.361}

Background Intentional injury (abuse) prevention efforts in sport can benefit from empirical data, but evidence delineating victim-, perpetrator-, and sport-setting-related risk factors over time, are limited. The U.S. Center for SafeSport, which houses a national-level database of disciplinary cases across American sports, provides opportunity to expand this evidence base.

Objective Identify abuse patterns in the U.S. Center for SafeSport Centralized Disciplinary Database (CDD).

Design Retrospective analysis of CDD cases. An alpha of 0.05 was used to test for significance.

Setting SafeSport CDD.

Patients (or Participants) 1164 CDD cases issued between January 1st, 1980 and January 16th, 2020.

Interventions (or Assessment of Risk Factors) Variables including year, sport, victim gender, team structure, uniform coverage, contact level, gender culture, gender structure, and subjective judging were analyzed, and their association with cases, identified.

Main Outcome Measurements Exploratory data analysis, with additional analyses on cases explicitly mentioning sexual harassment or abuse, or involvement of a minor. Comparisons were analyzed with chi-square.

Results 680 of 1164 cases were adjudicated in 2017 or later. 40 distinct sports were represented, including one Paralympic sport (Athletics). USA Gymnastics (217), Swimming (185), and Ice Hockey (110) had the most cases.

532 of 1164 cases mentioned sexual misconduct or sexual harassment. There was an association between this and individual sports $(\mathrm{p}<0.01)$, lower levels of clothing $(\mathrm{p}<0.01)$, non-contact sports $(p<0.01)$, gender culture $(p<0.01)$, and sports with subjective judging $(\mathrm{p}<0.01) .473$ out of 1164 cases mentioned the involvement of a minor. There was an 